\title{
Noise spectra of a semiconductor ring laser in the bidirectional regime
}

\author{
Antonio Pérez-Serrano, Roberta Zambrini, Alessandro Scirè, and Pere Colet \\ Instituto de Física Interdisciplinar y Sistemas Complejos, IFISC, CSIC-UIB, Campus UIB, E-07122 Palma de Mallorca, Spain
}

(Received 18 February 2009; published 30 October 2009)

\begin{abstract}
We analytically investigate the influence of complex backscattering coefficients and pump current on the noise spectra of a two-mode model for semiconductor ring laser in the Langevin formulation. The system features in the bidirectional regime are naturally described in terms of the two mode-intensity sum ( $I$ spectrum) and difference $(D$ spectrum). The $I$ spectrum reflects the energy exchange between the total field and the medium and behaves similarly to the relative intensity noise for single-mode semiconductor lasers. The $D$ spectrum represents the energy exchange between the two counterpropagating modes and is shaped by the noisy precursor of a Hopf bifurcation induced by the complex backscattering.
\end{abstract}

DOI: 10.1103/PhysRevA.80.043843

PACS number(s): 42.55.Px, 42.60.Mi, 42.65.-k

\section{INTRODUCTION}

Semiconductor ring lasers (SRLs) [1] have recently gained interest due to their peculiar properties from both fundamental and applicative points of view. As SRLs do not require claved facet mirrors or gratings for optical feedback, thus monolithic integration is easily achievable [2]. These devices exhibit different operating regimes characterized by bidirectional-continuous waves or alternate oscillations to bistability, mode locking, and chaos [3-7]. Whereas some regimes are shared with solid-state ring lasers (SSRLs) [8-10], SRLs possess peculiar features. In particular, the bistable regime is interesting for applications in integrated optical logics, optical gating, and reshaping [11], whereas the bidirectional regime has been widely exploited for rotation sensing applications [12].

An important issue in view of applications is about the effects of fluctuations in ring lasers as they change the performance as well as the dynamics of these devices. As a matter of fact, noise determines the performance of the ring laser gyroscope [13] and induces spontaneous switching in a bistable SRLs $[14,15]$. The main fundamental noise source of a semiconductor laser is represented by spontaneous emission, which yields to fluctuations in the signal intensity and frequency [16]. Different examples of how to model the spontaneous emission noise are shown in [17-21]. However, semiconductor ring lasers show peculiarities in the noise spectra that still deserve attention. New features in the modal power spectrum of a SRL were recently reported, such as the presence of an unexpected radio frequency peak explained in [22] as a mode partition noise effect associated to the intracavity backscattering.

While SRLs share some general characteristics with other ring lasers, they have some distinctive features such as coupling between phase and amplitude, which is known to affect phase noise [16], and strong intermodal gain cross saturation, which induces anticorrelated dynamics in the mode-power distribution $[3,30]$. Also, the light extraction system in integrated SRLs introduces a perturbation of the refraction index [23], which turns out to be a localized reflection enhancing the conservative backscattering. Therefore, SRLs experience conservative backscattering stronger than the dissipative one, differently from gas, dye [24], or solid-state ring lasers [10].
The influence of backscattering in the noise spectra constitutes a possible strategy to extract backscattering parameters in working conditions, useful for laser characterization.

In this paper, we consider the effects of the spontaneous emission noise in a two-mode rate equations model $[4,14,15,25,26]$ for a SRL operating in the bidirectional regime. In the following, after introducing the model, we review the operation regimes of SRLs by representation on a Poincaré sphere (Sec. II). We then focus on the bidirectional regime calculating analytically the linearized dynamics of the fluctuations and their correlations (Sec. III). Noise spectra are obtained for decoupled quantities that are modeintensity sum and difference. Correlations are calculated analytically within a linear approximation and compared to simulations of the full nonlinear model, showing a very good agreement in the considered regime.

\section{MODEL}

Considering single longitudinal-mode operation, the electric field inside the cavity reads

$$
E(x, t)=E_{+}(t) e^{-i(\Omega t-k x)}+E_{-}(t) e^{-i(\Omega t+k x)},
$$

where $E_{+}$and $E_{-}$are the mean-field slowly varying complex amplitudes of the electric field associated with the two propagation directions, $E_{+}$clockwise $(\mathrm{CW})$ and $E_{-}$counterclockwise $(\mathrm{CCW})$, respectively, being $x$ the spatial coordinate along the ring, assumed positive in the clockwise direction, and $\Omega$ is the optical frequency of the selected longitudinal mode. The model we consider is composed by the following set of dimensionless rate equations for the time evolution of the electric fields $E_{ \pm}$and the carrier density $N$ :

$$
\begin{aligned}
& \dot{E}_{ \pm}=\mathcal{G}_{ \pm}\left(N,\left|E_{ \pm}\right|^{2}\right) E_{ \pm}-\eta E_{\mp}+\xi_{ \pm}(t), \\
& \dot{N}=\gamma\left[\mu-N\left(1+\sigma_{+}\left|E_{+}\right|^{2}+\sigma_{-}\left|E_{-}\right|^{2}\right)\right],
\end{aligned}
$$

where (3) is the Bloch equation for the carrier dynamics,

$$
\mathcal{G}_{ \pm}\left(N,\left|E_{ \pm}\right|^{2}\right)=\frac{1}{2}(1+i \alpha)\left\{N \sigma_{ \pm}-1\right\}
$$

is the nonlinear gain, and 


$$
\sigma_{ \pm}=1-s\left|E_{ \pm}\right|^{2}-c\left|E_{\mp}\right|^{2},
$$

is the gain suppression function.

The complex backscattering coefficient is $\eta=k_{d}+i k_{c}$, with its dissipative $\left(k_{d}\right)$ and conservative $\left(k_{c}\right)$ components [27]. Dissipative backscattering is associated to light extraction losses, similarly to other RLs. On the other side, SRLs were experimentally found to exhibit strong conservative backscattering with respect to the dissipative one. This feature derives from unavoidable intracavity reflections at the light extraction region (evanescent coupler). In order to extract light, the curved waveguide is coupled to a straight waveguide by evanescent wave [23]. This introduces a localized perturbation to the effective refraction index which reverts to a source of localized back-reflections, i.e., conservative backscattering $[24,28]$. The values of the backscattering coefficients we take in this paper are extracted from experiments on real devices [4]. Also in Eq. (4), the $\alpha$ factor describes the phase-amplitude coupling mechanism present in semiconductor lasers. The saturation effects in the gain, written in the quadratic approximation, are represented by the parameters $s$ and $c$, which are normalized self- and crossgain saturation coefficients, respectively. The parameter $\mu$ represents the pump and is normalized to the threshold current (i.e., $\mu=1$ at threshold) and $\gamma$ is the ratio between the photon lifetime and the carrier lifetime. The model was proven to give excellent quantitative agreement with experiments $[3,4]$. The fluctuations terms $\xi_{ \pm}(t)$ are the Langevin forces [29], i.e., white Gaussian complex noise sources with non vanishing correlations

$$
\left\langle\xi_{ \pm}(t) \xi_{ \pm}^{*}\left(t^{\prime}\right)\right\rangle=2 \sqrt{\beta \tau_{p} N_{s t}} \delta\left(t-t^{\prime}\right),
$$

where $\tau_{p}$ is the photon lifetime, $N_{s t}$ is the carrier steady-state solution (11), and $\beta$ represents the fraction of spontaneously emitted photons coupled to the cavity. Noise terms $\xi_{+}$and $\xi_{-}$ reflect the effect of spontaneous emission in each direction of propagation. For simplicity, we do not take into account a noise source for the carrier density equation, considering the spontaneous emission as the main noise source in semiconductor lasers $[16,17]$.

According to the experimental fitting [3] through this paper, we take the following parameter sets (except where otherwise is noticed) $\alpha=3.5, s=0.005, c=0.01, k_{d}=3.27 \times 10^{-4}$, $k_{c}=4.4 \times 10^{-3}, \tau_{p}=10 \mathrm{ps}$, and $\gamma=2 \times 10^{-3}$.

\section{Instabilities of the SRL}

The bifurcation diagram of the SRL is obtained by numerical integration of Eqs. (2) and (3) and is shown in the first panel of Fig. 1 for increasing values of the pump coefficient $\mu$. As in all the regimes, the total intensity is constant and in order to analyze the evolution of the intensity difference and the relative phase between the two counterpropagating fields, we introduce new variables represented in a Poincaré sphere, the relative intensity $\theta$, and the relative phase $\psi$,

$$
\theta=2 \arctan \left(\frac{\left|E_{+}\right|^{2}-\left|E_{-}\right|^{2}}{\left|E_{+}\right|^{2}+\left|E_{-}\right|^{2}}\right),
$$

(1)

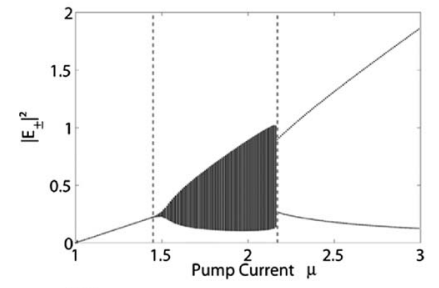

(3) $\mu=2$

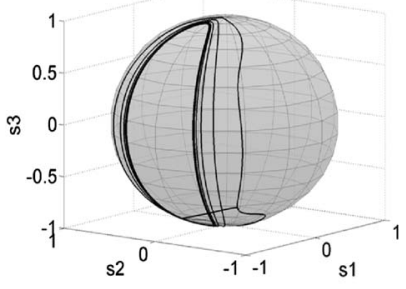

(2)

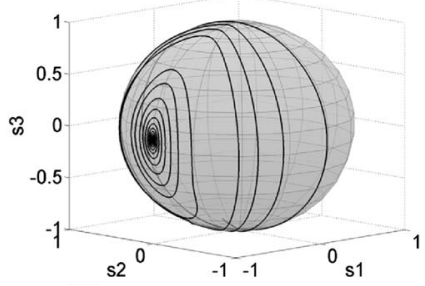

(4) $\mu=2.6$

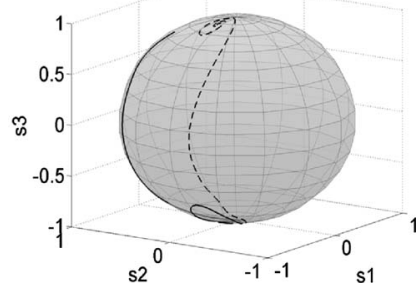

FIG. 1. (1) Bifurcation diagram for electric fields intensity for increasing pump current $\mu$. [(2)-(4)] Evolution of the Stokes parameters on the Poincaré spheres for each regime. (2) $\mu=1.2$, (3) $\mu=2$, and (4) $\mu=2.6$.

$$
\psi=\arctan \left(\frac{\operatorname{Im}\left(\frac{E_{+}}{E_{-}}\right)}{\operatorname{Re}\left(\frac{E_{+}}{E_{-}}\right)}\right) .
$$

In this context, $\theta$ is equivalent to ellipticity in the description of the polarization of the electric field in the sense that it describes how the field is distributed between the two counterpropagating modes (here analogous to the circular polarization modes). Those new variables are projected in the Poincaré sphere by means of the Stokes parameters as follows:

$$
\begin{gathered}
s 1=\cos \theta \cos \psi, \\
s 2=\cos \theta \sin \psi, \\
s 3=\sin \theta .
\end{gathered}
$$

We note that we consider a fixed value of the total intensity giving a sphere with unitary radius. The poles represent unidirectional solutions, being the north pole a pure $\mathrm{CW}$ operation and the south pole a pure $\mathrm{CCW}$ operation. The equator is the line where the two counterpropagating waves have the same intensity but different relative phases [31]. Along the equator, the point $s 2=0, s 3=0$ represents the two fields in phase while the point $s 2=1, s 3=0$ represents the two fields with a phase difference equal to $\pi$.

We can now visualize the instabilities of the SRL on the Poicaré sphere by numerical integration of Eqs. (2) and (3) [35]. Above threshold $(\mu>1)$, the first stable regime found is a symmetric solution (bidirectional regime), where the two counterpropagating fields have the same intensity. In panel (2) of Fig. 1, we see a trajectory starting from random initial conditions and ending at the fixed point $s 2=0$ in the equator. Increasing the pump $\mu$, a Hopf bifurcation takes place which 
marks the beginning of a limit cycle regime (alternate oscillations in $[4,10])$, where the two field intensities are oscillating in anticorrelated fashion, i.e., when one field reaches its maximum intensity, the other reaches its minimum and vice versa. Panel (3) shows the limit cycle on the Poincaré sphere reached from a random initial condition. Increasing further $\mu$, an asymmetric solution regime due to a pitchfork bifurcation is found [25]. Here, the two counterpropagating fields have different intensities and the difference increases with $\mu$. In this regime, depending on the initial conditions, the laser emission is mainly concentrated either in $\mathrm{CW}$ or $\mathrm{CCW}$ direction. Panel (4) shows two trajectories from different initial conditions and how they are attracted by different fixed points corresponding to the two emission directions. When $\mu$ increases, the two fixed points move toward the poles.

\section{FLUCTUATIONS DYNAMICS AND CORRELATIONS}

\section{A. Monochromatic solutions}

Focusing on the bidirectional regime, we look for a monochromatic solution for the two fields with the same amplitude

$$
E_{ \pm}(t)=Q e^{i \omega t \pm i \phi} .
$$

SRLs admit two possible solutions. In the in-phase case,

$$
\phi_{\text {in }}=0 \rightarrow \omega_{\text {in }}=\alpha k_{d}-k_{c},
$$

while in the out-of-phase case,

$$
\phi_{\text {out }}=\frac{\pi}{2} \rightarrow \omega_{\text {out }}=-\alpha k_{d}+k_{c}
$$

Depending on the sign of the backscattering parameters, one of the solutions is stable and the other unstable. If $k_{d}>0\left(k_{d}<0\right)$, the out-of-phase case is stable (unstable). Physically, the two solutions are equivalent because the sign of $k_{d}$ represent which direction [clockwise $(\mathrm{CW})$ or counterclockwise $(\mathrm{CCW})]$ is chosen as positive. From now on, we focus on the out-of-phase case without loosing generality. The corresponding stationary solution for the carrier density $N=N_{s t}$ as a function of the amplitude of the fields and the pump parameter is

$$
N_{s t}=\frac{\mu}{1+2 Q^{2} f},
$$

where $f=1-s Q^{2}-c Q^{2}$. For the amplitude $Q$, we find

$$
Q^{2}=\frac{N_{s t}-1+k_{d}}{(c+s) N_{s t}} .
$$

\section{B. Linear fluctuations dynamics}

Hereby, we analyze the effect of a perturbation on the stationary solutions. We consider a real perturbation $n$ in the carrier density and complex perturbations $a_{ \pm}$for the fields

$$
\begin{gathered}
E_{ \pm}=\left(Q+a_{ \pm}\right) e^{i \omega t \pm i \phi}, \\
N=N_{s t}+n .
\end{gathered}
$$

By making use of Eq. (13) in Eqs. (2) and (3), we derive the following linear system:

$$
\begin{aligned}
\dot{n}= & -\gamma\left\{\left[1-2 Q^{2}(s+c)\right] N_{s t} Q\left(a_{+}+a_{+}^{*}+a_{-}+a_{-}^{*}\right)\right. \\
& \left.+\left(1+2 Q^{2} f\right) n\right\}, \\
\dot{a}_{ \pm}= & \frac{1}{2}(1+i \alpha)\left\{\left(N_{s t} f-1\right) a_{ \pm}\right. \\
& \left.-N_{s t} Q^{2}\left[s\left(a_{ \pm}+a_{ \pm}^{*}\right)+c\left(a_{\mp}+a_{\mp}^{*}\right)\right]+Q f n\right\}-i \omega a_{ \pm} \\
& -\eta(\cos 2 \phi \mp i \sin 2 \phi) a_{\mp}+\xi_{ \pm} .
\end{aligned}
$$

At this point, we introduce a new set of variables to simplify the set (14) in two independent problems by block diagonalization. The new variables are

$$
\begin{aligned}
& S=a_{+}+a_{-}, \\
& R=a_{+}-a_{-} .
\end{aligned}
$$

We can relate those new variables to the typical experimentally measured quantities $\left|E_{+}\right|^{2}$ and $\left|E_{-}\right|^{2}$ defining

$$
\begin{aligned}
& \mathbb{I}=\left|E_{+}\right|^{2}+\left|E_{-}\right|^{2}, \\
& \mathbb{D}=\left|E_{+}\right|^{2}-\left|E_{-}\right|^{2},
\end{aligned}
$$

and writing those new variables as $\mathbb{I}=I_{0}+I$ and $\mathrm{D}=D_{0}+D$, where $I_{0}$ and $D_{0}$ are constants and the perturbations, $I$ and $D$ can be expressed in terms of $S$ and $R$ at first order,

$$
\begin{gathered}
I=Q\left(S+S^{*}\right), \\
D=Q\left(R+R^{*}\right) .
\end{gathered}
$$

The variable $I$ describes the perturbation of the total intensity of the laser regardless of its distribution between the two modes, whereas $D$ describes the power exchange between the two counterpropagating fields.

\section{Relative Intensity}

The equation for the dynamic evolution of $R$ is

$$
\dot{R}=(1+i \alpha) K\left(R+R^{*}\right)-2 \eta R+\xi_{R}(t),
$$

where the fluctuation term is derived from Eqs. (15) and (5), $\xi_{R}(t)=\xi_{+}(t)-\xi_{-}(t)$ with the correlation properties

$$
\left\langle\xi_{R}(t) \xi_{R}^{*}\left(t^{\prime}\right)\right\rangle=4 \sqrt{\beta \tau_{p} N_{s t}} \delta\left(t-t^{\prime}\right),
$$

and $K$ is a real constant defined by

$$
K=\frac{1}{2} N_{s t} Q^{2}(c-s) .
$$

The corresponding eigenvalues for the differential equations system for $R$ and $R^{*}$ are

$$
\lambda_{1,2}=K-2 k_{d} \pm\left[K^{2}+4 K \alpha k_{c}-4 k_{c}^{2}\right]^{1 / 2} .
$$

The above eigenvalues are used to construct the stability diagrams shown in Fig. 2 as functions of the two backscattering parameters. The figure shows how above a certain threshold for the conservative backscattering $k_{c}$ the eigenvalues become complex conjugate (vertical line in all panels in Fig. 

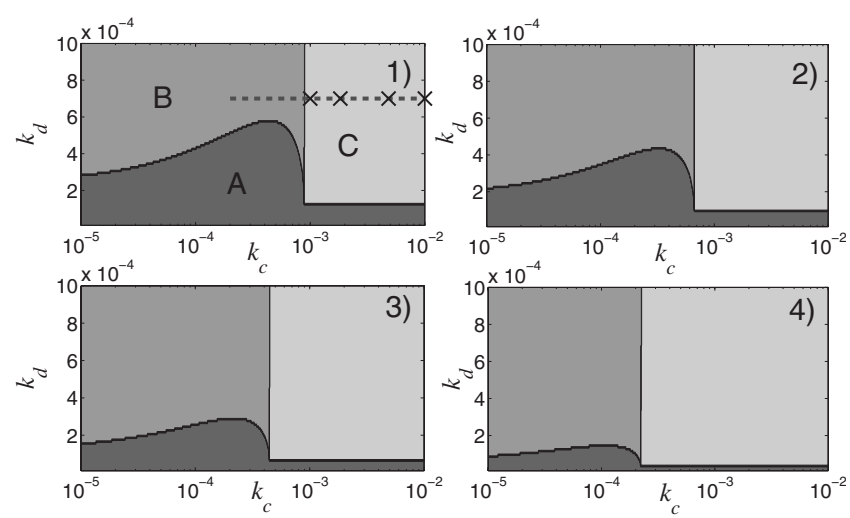

FIG. 2. Stability diagrams of the symmetric solutions (10)-(12) for different values of $\mu$ depending on backscattering coefficients. In region $\mathrm{A}$, the symmetric solution is unstable, region $\mathrm{B}$ is stable with real eigenvalues, and in region $\mathrm{C}$ is stable with complex conjugates eigenvalues. (1) corresponds to $\mu=1.2$, (2) to $\mu=1.15$, (3) to $\mu=1.1$, and (4) to $\mu=1.05$.

2). This implies the presence of a new eigenfrequency in the system. In the unstable region $\mathrm{A}$, such frequency originates a limit cycle, according to previous works [4], whereas in regions $\mathrm{B}$ and $\mathrm{C}$, perturbations relax to the monochromatic solution. For increasing values of the pump $\mu$, the unstable region A becomes less pronounced.

The two-mode difference at a given frequency reads as

$$
\begin{aligned}
\tilde{R}(\omega)= & \frac{1}{A(\omega)}\left\{\left[i \omega-(1-i \alpha) K+2 \eta^{*}\right] \widetilde{\xi}_{R}(\omega)+(1+i \alpha) \widetilde{K \xi_{R}^{*}}(\right. \\
& -\omega)\},
\end{aligned}
$$

where

$$
A(\omega)=-\omega^{2}+i \omega\left(4 k_{d}-2 K\right)-4\left(k_{d}+\alpha k_{c}\right) K+4\left(k_{d}^{2}+k_{c}^{2}\right),
$$

leading to the two-frequency correlation

$$
\begin{aligned}
\left\langle\widetilde{R}(\omega) \widetilde{R}^{*}\left(\omega^{\prime}\right)\right\rangle= & \frac{1}{A(\omega) A(-\omega)}\left[4 k_{d}^{2}-4 K\left(k_{d}+k_{c} \alpha\right)\right. \\
& +2 K^{2}\left(1+\alpha^{2}\right)+2 K \alpha \omega \\
& \left.+\left(\omega-2 k_{c}\right)^{2}\right] 8 \pi \sqrt{\beta \tau_{p} N_{s t}} \delta\left(\omega-\omega^{\prime}\right) .
\end{aligned}
$$

From Eq. (19), we immediately obtain the $D$ spectrum

$$
\left\langle\widetilde{D}(\omega) \widetilde{D}^{*}\left(\omega^{\prime}\right)\right\rangle=Q^{2}\left(\left\langle\widetilde{R}(\omega) \widetilde{R}^{*}\left(\omega^{\prime}\right)\right\rangle+\left\langle\widetilde{R}(-\omega) \widetilde{R}^{*}\left(-\omega^{\prime}\right)\right\rangle\right) .
$$

The analytical result is shown in Fig. 3 for a parameter set in region $\mathrm{C}$ of Fig. 2. The analytic is compared to numerical simulations of the full nonlinear system [36]. Physically, the backscattering represents the energy exchange rate between the two modes. Such process shows a resonance (the peaks in Fig. 3-5) which is more evident for increasing values of gain cross-saturation and conservative backscattering, whereas the resonance is damped for increasing values of gain self-saturation and dissipative backscattering (see continuous black line in Fig. 4).

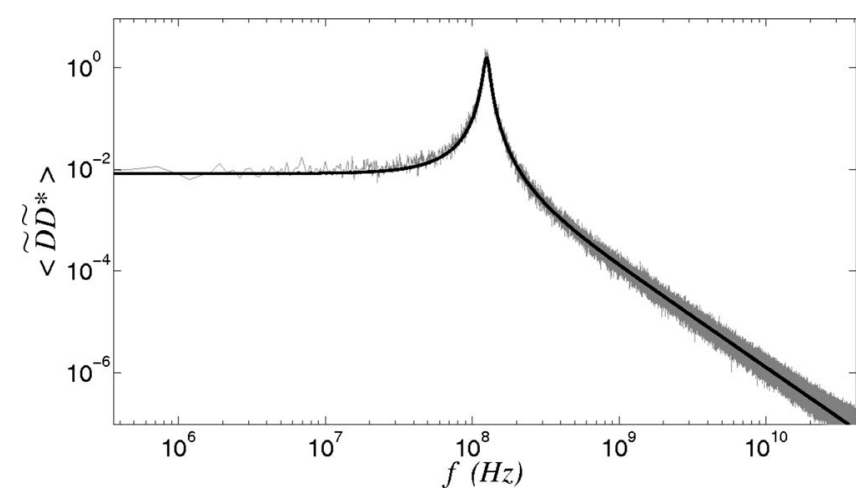

FIG. 3. $D$ spectrum. The gray line corresponds to the numerical simulation for 20 noise realizations; the analytical solution is the black line. $\beta=10^{-3} \mathrm{~ns}^{-1}, \alpha=3.5, s=0.005, \quad c=0.01$, $k_{d}=3.27 \times 10^{-4}, k_{c}=4.4 \times 10^{-3}, \gamma=2 \times 10^{-3}$, and $\mu=1.2$. The frequency $f$ is found from the adimensional angular frequency $\omega$. Here and in the following figures, $f=\frac{\omega}{2 \pi \tau_{p}}$, where $\tau_{p}=10 \mathrm{ps}$ is the photon lifetime in the ring cavity.

SRLs are well modeled by strong cross-saturation and conservative backscattering. For such parameter choices, our study unveils the presence of a resonance peak in the frequency spectrum. Such behavior was reported in a recent experimental work [22].

Figure 4 shows how the $D$ spectrum is modified by conservative backscattering coefficient using the analytical expression (25) for parameter values corresponding to the crosses in Fig. 2. The figure shows that the peak is more pronounced when moving deeper into region C. Backscattering parameters are difficult to measure in operating conditions and they are important to determine the static and dynamic properties of the laser. Such noise spectra, corresponding to measurements of the correlation spectrum of the intensity difference fluctuations, represent a novel and suitable way to extract the actual extent of the backscattering in its dissipative and conservative parts in working conditions. Figure 5 shows the $D$-spectrum dependence on pump current of the analytical expression (25) for pump values that

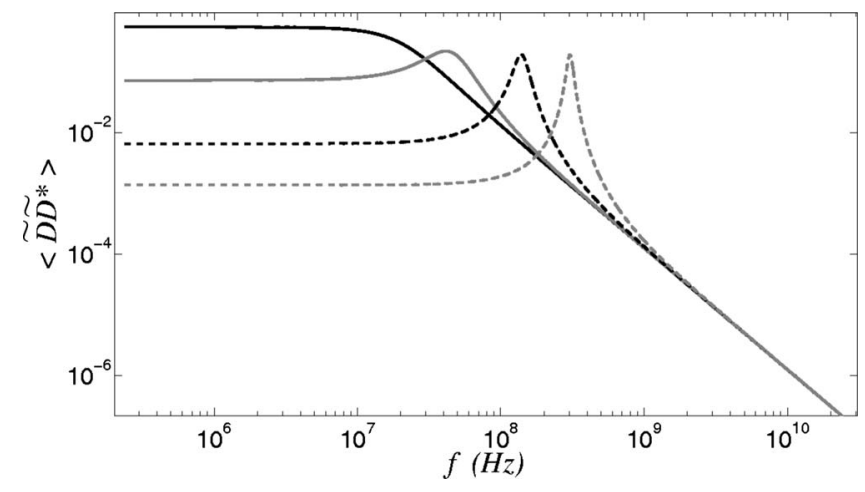

FIG. 4. $D$ spectrum. Dependence on conservative backscattering coefficient, $k_{c}$. Black curve corresponds to $k_{c}=10^{-3}$, the gray curve to $k_{c}=1.83 \times 10^{-3}$, the dashed black curve to $k_{c}=4.83 \times 10^{-3}$, and the dashed gray curve to $k_{c}=10^{-2}$. Each spectrum corresponds each cross in panel (1) in Fig. $2 ; \beta=10^{-3} \mathrm{~ns}^{-1}, k_{d}=4 \times 10^{-4}$, and $\mu=1.2$. 


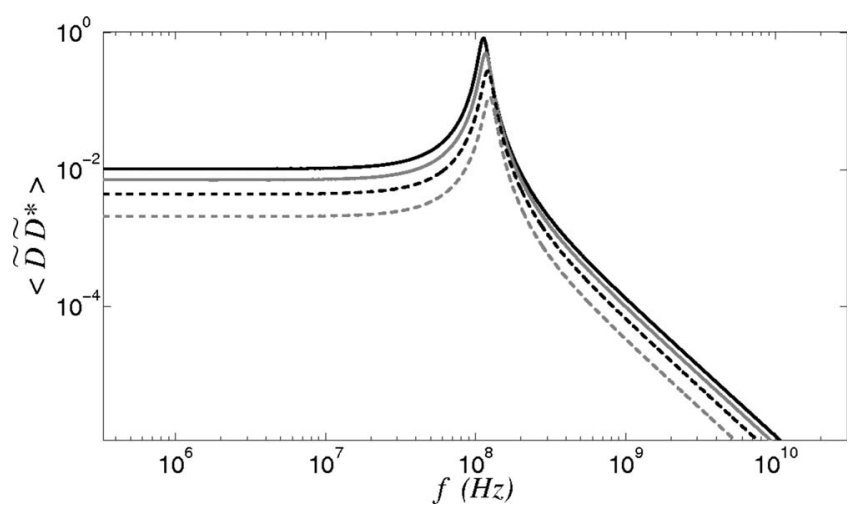

FIG. 5. $D$-spectrum dependence on pump current $\mu$. Black curve corresponds to $\mu=1.2$, the gray curve to $\mu=1.15$, the dashed black curve to $\mu=1.1$, and the dashed gray curve to $\mu=1.05$; $\beta=10^{-3} \mathrm{~ns}^{-1}, k_{c}=4 \times 10^{-3}$, and $k_{d}=4 \times 10^{-4}$.

maintain the SRL in the bidirectional regime. The figure shows the persistence of the resonance peak for increasing pumps.

In time domain, such resonance emerges as a consequence of the system oscillating around the fixed point due to spontaneous emission noise. Figure 6 shows the evolution of $\theta$ and $\psi$ for the complete set (2) and (3) including spontaneous emission noise in the bidirectional regime for different $\beta$ values. In the absence of noise [see Fig. 1 panel (2)], the variables $\theta$ and $\psi$ end in the fixed point after a transient, but in the presence of noise, the transient becomes longer depending on the value of $\beta$ and produces slow undamped oscillations around the fixed point. This is reported in literature as Hopf bifurcation precursor [32]. Therefore, in our interpretation, the radio frequency resonance peak experimentally [22] observed is given by a noise driven excitation near a fixed point.

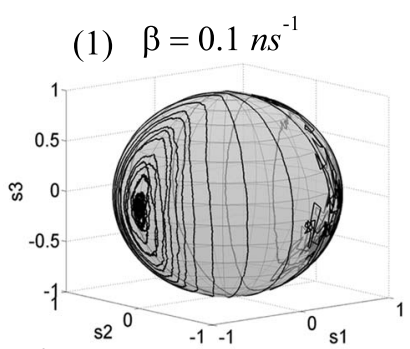

(2) $\beta=10^{-3} n s^{-1}$

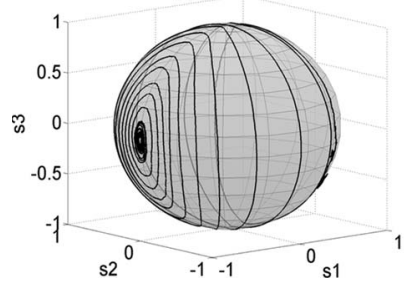

(3) $\beta=10^{-5} n s^{-1}$

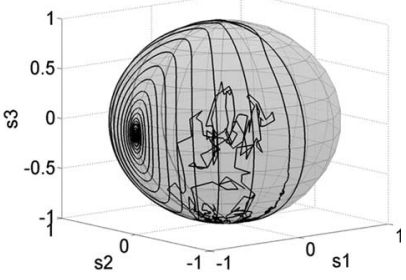

FIG. 6. Poincaré spheres for the bidirectional regime in the presence of noise. (1) $\beta=0.1 \times \mathrm{ns}^{-1}$, (2) $\beta=10^{-3} \mathrm{~ns}^{-1}$, and (3) $\beta=10^{-5} \mathrm{~ns}^{-1}$. $\mu=1.3$.

\section{Total intensity and carrier density}

The equations for the dynamic evolution of $S$ and $n$ are

$$
\begin{aligned}
\dot{S} & =(1+i \alpha)\left\{C n+\widetilde{K}\left(S+S^{*}\right)\right\}+\xi_{S}(t), \\
\dot{n}= & -\gamma\left\{n+\left[1-2 Q^{2}(s+c)\right] N_{s t} Q\left(S+S^{*}\right)\right. \\
& \left.+2 Q^{2}\left[1-s Q^{2}-c Q^{2}\right] n\right\},
\end{aligned}
$$

where the fluctuation term is derived from Eqs. (15) and (5), $\xi_{S}(t)=\xi_{+}(t)+\xi_{-}(t)$, with the same correlation properties shown in the previous section (21) and $\widetilde{K}$ and $C$ are real constants

$$
\begin{gathered}
\tilde{K}=-\frac{1}{2} N_{s t} Q^{2}(c+s), \\
C=Q\left[1-Q^{2}(c+s)\right] .
\end{gathered}
$$

The corresponding eigenvalues for the system (27) are

$$
\lambda_{0}=0,
$$

$$
\begin{aligned}
\lambda_{1,2}= & \tilde{K}-\frac{\gamma}{2}-\gamma Q C \pm \frac{1}{2}\left\{\gamma^{2}+4\left[\tilde{K}^{2}+\widetilde{K} \gamma+\gamma^{2} Q C(1+Q C)\right]\right. \\
& \left.-8 \gamma\left(\tilde{K} Q C+N_{s t} C^{2}\right)\right\}^{1 / 2} .
\end{aligned}
$$

The presence of a zero eigenvalue indicates that the system (27) is singular. The corresponding neutral eigenmode, known as Goldstone mode [34], appears because the solution in the bidirectional regime breaks the global phase invariance and gives rise to large undamped fluctuations. Indeed, the Goldstone mode is associated to the imaginary part of $S$. Interestingly, by using Eq. (18), we can get rid of this mode by decoupling the fluctuations dynamics from it and reducing the dynamics to a subspace orthogonal to the Goldstone mode itself. Then a linear approximation is well-justified in terms of the variable $I$, therefore the equation system (27) reads

$$
\begin{gathered}
\dot{I}=2 \widetilde{K} I+2 Q C n+\xi_{I}(t), \\
\dot{n}=-\gamma\left[1-2 Q^{2}(s+c)\right] N_{s t} I-\gamma(1+2 Q C) n,
\end{gathered}
$$

where the fluctuation term is derived from Eqs. (15) and (5), $\xi_{I}(t)=Q \operatorname{Re}\left[\xi_{S}(t)+\xi_{S}^{*}(t)\right]$, with the correlation properties

$$
\left\langle\xi_{I}(t) \xi_{I}\left(t^{\prime}\right)\right\rangle=\left\langle\xi_{I}(t) \xi_{I}^{*}\left(t^{\prime}\right)\right\rangle=8 Q^{2} \sqrt{\beta \tau_{p} N_{s t}} \delta\left(t-t^{\prime}\right) .
$$

By Fourier transform, we derive

$$
\widetilde{I}(\omega)=\frac{1}{B(\omega)}[i \omega+\gamma(1+2 Q C)] \tilde{\xi}_{I}(\omega)
$$

and

$$
\widetilde{n}(\omega)=\frac{-1}{B(\omega)} \gamma N_{s t}\left[1-2 Q^{2}(s+c)\right] \widetilde{\xi}_{I}(\omega),
$$

where 


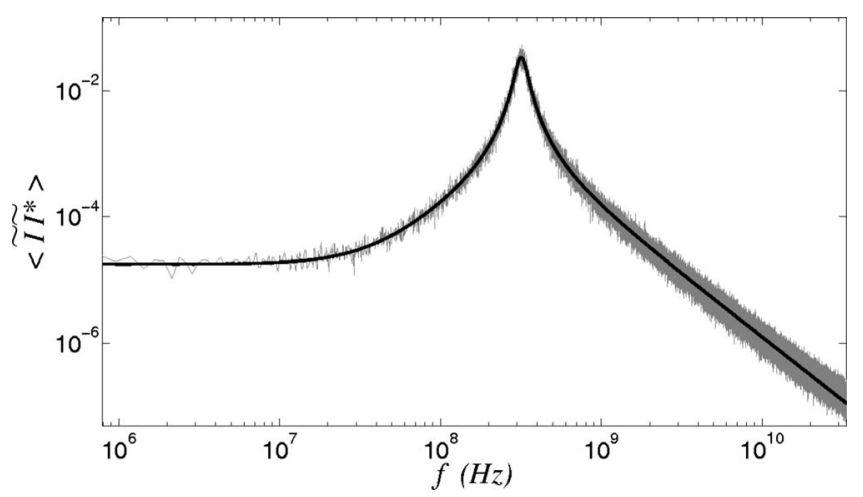

FIG. 7. I spectrum vs frequency. Gray line corresponds to the numerical simulation and black line is the analytical solution (35), $\beta=10^{-3} \mathrm{~ns}^{-1}$.

$$
\begin{aligned}
B(\omega)= & -\omega^{2}+i \omega[\gamma(1+2 Q C)-2 \tilde{K}] \\
& +2 \gamma\left[Q C N_{s t}+\tilde{K}(2 Q-1)\right]
\end{aligned}
$$

We are able to find the following ensemble average:

$$
\begin{aligned}
\left\langle\widetilde{I}(\omega) \widetilde{I^{*}}\left(\omega^{\prime}\right)\right\rangle= & \frac{Q^{2}}{\widetilde{B}(\omega) \widetilde{B}^{*}(\omega)} \\
& \times\left[\omega^{2}+\gamma^{2}(1+2 Q C)^{2}\right] 16 \pi \sqrt{\beta \tau_{p} N_{s t}} \delta\left(\omega-\omega^{\prime}\right) .
\end{aligned}
$$

Figure 7 shows the good agreement between $I$ spectrum from Eq. (35) and numerical simulations. The observed peak corresponds to the typical relaxation oscillations of the fieldmedium-energy exchange process and its frequency depends on the pump and medium characteristics. The backscattering does not play any role in the field-medium-energy exchange process as can be seen from Eq. (35).

\section{CONCLUSIONS}

We have studied the influence of spontaneous emission noise in a two-mode model for semiconductor ring lasers biased in the bidirectional regime. The analysis has been carried out by linearizing the model close to a stable stationary solution and considering the effect of spontaneous emission as stochastic perturbations expressed by Langevin forces. At a linear level, perturbations concerning the total intensity and carrier inversion dynamics decouple from the energy distribution processes between the two modes. This fact has permitted a full analytic analysis, well confirmed by numerical simulations of the complete non linear system. The analysis showed that semiconductor ring lasers have peculiar noise properties. On one side, the total intensity and carrier density show a noise spectrum (I spectrum) characterized by a resonance induced by the typical field-mediumenergy exchange process (relaxation oscillations) and the global phase diffusion induced by the Goldstone mode. Besides, the degree of freedom associated to the simultaneous presence of two counterpropagating modes allows for a further process of energy exchange between the two modes. Our analysis unveiled that such process presents a resonance influenced mainly by the backscattering parameters and interpreted as a "noisy precursor" of a Hopf bifurcation. This opens the possibility to extract the backscattering parameters from the noise spectra.

\section{ACKNOWLEDGMENTS}

This work was funded by the European project IOLOS Project No. IST-2005-34743 and Spanish projects PhoDeCC Project No. TEC2006-10009/MIC, FISICOS Project No. FIS2007-60327, and ECusCo Project No. 2008501047. A.S. acknowledges Ramón y Cajal program by Spanish MEC.
[1] A. S. H. Liao and S. Wang, Appl. Phys. Lett. 36, 801 (1980).

[2] T. Krauss, P. J. R. Laybourn, and J. S. Roberts, Electron. Lett. 26, 2095 (1990).

[3] M. Sorel, A. Scirè, G. Giuliani, R. Miglierina, S. Balle, P. J. R. Laybourn, and S. Donati, Opt. Lett. 27, 1992 (2002).

[4] M. Sorel, G. Giuliani, A. Scirè, R. Miglierina, S. Donati, and P. J. R. Laybourn, IEEE J. Quantum Electron. 39, 1187 (2003).

[5] M. Sorel, P. J. R. Laybourn, G. Giuliani, and S. Donati, Appl. Phys. Lett. 80, 3051 (2002).

[6] C. Born, M. Sorel, and S. Yu, IEEE J. Quantum Electron. 41, 261 (2005).

[7] H. Nakatsuka, S. Asaka, H. Itoh, K. Ikeda, and M. Matsuoka, Phys. Rev. Lett. 50, 109 (1983).

[8] P. A. Khandokhin and Ya. I. Khanin, J. Opt. Soc. Am. B 2, 226 (1985).

[9] I. I. Zolotoverkh, N. V. Kravtsov, E. G. Lariontsev, A. A. Makarov, and V. V. Firsov, Opt. Commun. 113, 249 (1994).

[10] N. V. Kravtsov and E. G. Lariontsev, IEEE J. Quantum Electron. 36, 192 (2006).
[11] M. T. Hill, H. J. S. Dorren, T. de Vrie, X. J. M. Leijtens, J. H. den Besten, B. Smalbrugge, Y. S. Oei, H. Binsma, G. D. Khoe, and M. K. Smit, Nature (London) 432, 206 (2004).

[12] S. Donati, Electro-Optical Instrumentation (Prentice Hall, Englewood Cliffs, NJ, 2004).

[13] W. W. Chow, J. Gea-Banacloche, L. M. Pedrotti, V. E. Sanders, W. Schleich, and M. O. Scully, Rev. Mod. Phys. 57, 61 (1985).

[14] T. Pérez, A. Scirè, G. Van der Sande, P. Colet, and C. R. Mirasso, Opt. Express 15, 12941 (2007).

[15] S. Beri, L. Gelens, M. Mestre, G. Van der Sande, G. Verschaffelt, A. Scirè, G. Mezosi, M. Sorel, and J. Danckaert, Phys. Rev. Lett. 101, 093903 (2008).

[16] K. Petermann, Laser Diode Modulation and Noise (Kluwer Academic Publishers, Dordrecht, 1988).

[17] M. San Miguel and R. Toral, Stochastic Effects in Physical Systems, Instabilities and Nonequilibrium Structures VI, edited by E. Tirapegui, J. Martínez, and R. Tiemann (Kluwer Academic Publishers, Dordrecht, 2000), pp. 35-130. 
[18] S. Balle, P. Colet, and M. San Miguel, Phys. Rev. A 43, 498 (1991).

[19] S. Balle, F. de Pasquale, N. B. Abraham, and M. San Miguel, Phys. Rev. A 45, 1955 (1992).

[20] F. Pedaci, S. Lepri, S. Balle, G. Giacomelli, M. Giudici, and J. R. Tredicce, Phys. Rev. E 73, 041101 (2006).

[21] H. Haug and S. W. Koch, Quantum Theory of the Optical and Electronic Properties of Semiconductors (World Scientific, Singapore, 2004).

[22] C. Ji, M. F. Booth, A. T. Schremer, and J. M. Ballantyne, IEEE J. Quantum Electron. 41, 925 (2005).

[23] S. Furst, A. Pérez-Serrano, A. Scirè, M. Sorel, and S. Balle, Appl. Phys. Lett. 93, 251109 (2008).

[24] C. Etrich, P. Mandel, R. Centeno Neelen, R. J. C. Spreeuw, and J. P. Woerdman, Phys. Rev. A 46, 525 (1992).

[25] G. Van der Sande, L. Gelens, Ph. Tassin, A. Scirè, and J. Danckaert, J. Phys. B 41, 095402 (2008).

[26] L. Gelens, S. Beri, G. Van der Sande, J. Danckaert, N. Calabretta, H. J. S. Dorren, R. Notzel, A. J. M. Bente, and M. K. Smit, Opt. Express 16, 10968 (2008).

[27] H. A. Haus, H. Statz, and I. W. Smith, IEEE J. Quantum Elec- tron. 21, 78 (1985).

[28] R. J. C. Spreeuw, R. C. Neelen, N. J. van Druten, E. R. Eliel, and J. P. Woerdman, Phys. Rev. A 42, 4315 (1990).

[29] C. W. Gardiner, Handbook of Stochastic Methods for Physics, Chemistry and the Natural Sciences, 2nd ed. (Springer-Verlag, Berlin, 1990), p. 107.

[30] G. Giuliani, R. Miglierina, M. Sorel, and A. Scirè, IEEE J. Sel. Top. Quantum Electron. 11, 1187 (2005).

[31] G. P. Agrawal, Phys. Rev. A 37, 2488 (1988).

[32] K. Wiesenfeld, Phys. Rev. A 32, 1744 (1985).

[33] R. Toral and A. Chakrabarti, Comput. Phys. Commun. 74, 327 (1993).

[34] D. Foster, Hydrodynamic Fluctuations, Broken Symmetry, and Correlation Fluctuations (Addison-Wesley, Redwood City, CA, 1983).

[35] Here, noise terms are neglected and we use a fourth-order Runge-Kutta algorithm with time discretization $\delta t=10^{-2}$.

[36] Numerical simulations of the stochastic differential equations (2) and (3) performed with a second-order Heun algorithm [17] and with random numbers generator described in [33]. 